\title{
Regularization and preconditioning of KKT systems arising in nonnegative least-squares problems
}

\author{
Stefania Bellavia ${ }^{1}$, Jacek Gondzio ${ }^{2}$ and Benedetta Morini ${ }^{1, *, \dagger}$ \\ ${ }^{1}$ Dipartimento di Energetica 'S. Stecco', Università di Firenze, via C. Lombroso 6/17, 50134 Firenze, Italy \\ ${ }^{2}$ School of Mathematics, The University of Edinburgh, Mayfield Road, Edinburgh EH9 3JZ, Scotland, U.K.
}

\begin{abstract}
SUMMARY
A regularized Newton-like method for solving nonnegative least-squares problems is proposed and analysed in this paper. A preconditioner for KKT systems arising in the method is introduced and spectral properties of the preconditioned matrix are analysed. A bound on the condition number of the preconditioned matrix is provided. The bound does not depend on the interior-point scaling matrix. Preliminary computational results confirm the effectiveness of the preconditioner and fast convergence of the iterative method established by the analysis performed in this paper. Copyright (c) 2008 John Wiley \& Sons, Ltd.
\end{abstract}

Received 30 August 2007; Revised 5 May 2008; Accepted 6 May 2008

KEY WORDS: bound-constrained linear least-squares problems; inexact Newton methods; iterative linear solvers; KKT systems; regularization; preconditioning

\section{INTRODUCTION}

Optimization problems having a least-squares objective function along with simple constraints arise naturally in image processing, data fitting, control problems and intensity-modulated radiotherapy problems. In the case where only one-sided bounds apply, there is no lack of generality to consider the nonnegative least-squares (NNLS) problems:

$$
\min _{x \geqslant 0} q(x)=\frac{1}{2}\|A x-b\|_{2}^{2}
$$

where $A \in \mathbb{R}^{m \times n}, b \in \mathbb{R}^{m}$ are given and $m \geqslant n[1]$.

\footnotetext{
*Correspondence to: Benedetta Morini, Dipartimento di Energetica 'S. Stecco', Università di Firenze, via C. Lombroso 6/17, 50134 Firenze, Italy.

${ }^{\dagger}$ E-mail: benedetta.morini@unifi.it

Contract/grant sponsor: GNCS/INDAM, Italy
}

Copyright (C) 2008 John Wiley \& Sons, Ltd. 
We assume that $A$ has full column rank so that the NNLS problem (1) is a strictly convex optimization problem and there exists a unique solution $x^{*}$ for any vector $b$ [2]. We allow the solution $x^{*}$ to be degenerate, that is, strict complementarity may not hold at $x^{*}$. We are concerned with the situation when $m$ and $n$ are large and we expect matrix $A$ to be sparse. We address the issues of the numerical solution of (1) and apply the interior-point Newton-like method given in [3] to it. From numerical linear algebra perspective this method can be reduced to solving a sequence of (unconstrained) weighted least-squares subproblems. Following [3] the linear systems involved have the following form:

$$
\left(\begin{array}{cc}
I & A S \\
S A^{\mathrm{T}} & -W E
\end{array}\right)\left(\begin{array}{l}
\tilde{q} \\
\tilde{p}
\end{array}\right)=\left(\begin{array}{l}
d \\
0
\end{array}\right)
$$

where $S, W, E$ are diagonal matrices satisfying $S^{2}+W E=I$ and matrix $W E$ is positive semidefinite. Bellavia et al. [3] solve this system with an iterative method, which corresponds to the use of an inexact Newton-like method to solve NNLS. The reader interested in the convergence analysis of this method should consult $[3,4]$ and the references therein.

In this paper we go a step further. System (2) arising in interior-point method [3] is potentially very ill-conditioned and therefore may be difficult to solve with iterative methods. Although preconditioning can help to accelerate the convergence of the iterative method, in some situations it is insufficient to ensure fast convergence. We remedy it in this paper by regularizing (2) to guarantee that the condition number of the matrix involved is decreased. Next, we design a preconditioner for such regularized systems and analyse the spectral properties of the preconditioned matrix.

The diagonal matrix $W E$ in (2) displays an undesirable property: certain elements of it may be null and others may be close to 1 . Inspired by the work of Saunders [5] and encouraging numerical experience reported by Altman and Gondzio [6] we regularize the $(2,2)$ block in (2) and replace element $W E$ with $W E+S^{2} \Delta$, where $\Delta$ is a diagonal matrix. We provide a detailed spectral analysis of the regularized matrix and prove that the condition number of the regularized system is significantly smaller than that of the original system. Following [6] we use a dynamic regularization, namely, we do not alter those elements of $W E$ that are bounded away from zero; we change only those that are too close to zero.

Having improved the conditioning of (2) we hope to have improved the rate of convergence of the applicable iterative methods. In fact, (2) is a saddle point problem with a simple structure [7]. We design an indefinite preconditioner that exploits the partitioning of indices into 'large' and 'small' elements in the diagonal of $W E$. The particular form of the regularization yields a relevant advantage: if the partitioning in $W E$ does not change from an iteration to another, then the factorization of the preconditioner is available for the new iteration at no additional cost. Moreover, as the algorithm approaches the optimal solution, the partitioning settles down following the splitting of indices into those corresponding to active and inactive constraints. Then, eventually the factorization of the preconditioner does not require computational effort.

We provide the spectral analysis of the preconditioned system and show that the condition number of its matrix is bounded by a number that does not depend on interior-point scaling. We are not aware of any comparable result in the literature. Moreover, the proposed preconditioner allows us to use the short recurrence projected preconditioned conjugate gradient (PPCG) method [8] to solve the preconditioned linear system. Thus, we also performed the spectral analysis of the reduced preconditioned normal equation whose eigenvalues determine the convergence of the PPCG method. Our preliminary computational results confirm all theoretical findings. Indeed, we 
have observed that the augmented system can be solved very efficiently by the PPCG method. For the medium- and large-scale problems (reaching a couple of hundred thousand constraints) iterative methods converge in a low number of iterations.

Regularization adds a perturbation to the Newton system. We prove that under suitable conditions on the regularization it does not worsen the convergence properties of the Newton-like method of Bellavia et al. [3]. Namely, the inexact regularized Newton-like method still enjoys $q$-quadratic convergence, even in presence of degenerate solutions.

We remark that our method can also be used to solve regularized least-squares problems:

$$
\min _{x \geqslant 0} q(x)=\frac{1}{2}\|A x-b\|_{2}^{2}+\mu\|x\|^{2}
$$

where $\mu$ is a strictly positive scalar. In this case, the arising augmented systems are regularized 'naturally' and we do not need to introduce the regularization of the $(2,2)$ block. Moreover, $A$ may also be rank deficient. Finally, the method can clearly handle lower and upper bounds on the variables too. We decided to limit our discussion to NNLS problems for the sake of simplicity.

This paper is organized as follows. In Section 2, we recall the key features of the Newton-like method studied in [3] and justify the need for introducing a regularization. In Section 3, we introduce the regularization and show that it does not worsen local convergence properties of the Newtonlike method. In Section 4, we analyse numerical properties of the regularized augmented system, introduce the preconditioner and perform spectral analysis of the preconditioned matrix. We also analyse the reduced normal equations formulation of the problem and for completeness perform spectral analysis of the preconditioned normal equations. In Section 5, we discuss preliminary computational results and in Section 6, we conclude our findings.

\subsection{Notations}

We use the subscript $k$ as index for any sequence and for any function $f$ we denote $f\left(x_{k}\right)$ by $f_{k}$. The symbol $x_{i}$ or $(x)_{i}$ denotes the $i$ th component of a vector $x$. The 2-norm is indicated by $\|\cdot\|$.

\section{THE NEWTON-LIKE METHOD}

In this section we briefly review the Newton-like method proposed in [3]. Then, we study the properties of the linear system arising at each iteration.

The Newton-like method in [3] is applied to the system of nonlinear equations:

$$
D(x) g(x)=0
$$

where $g(x)=\nabla q(x)=A^{\mathrm{T}}(A x-b)$, and $D(x)=\operatorname{diag}\left(d_{1}(x), \ldots, d_{n}(x)\right), x \geqslant 0$, has entries of the form

$$
d_{i}(x)= \begin{cases}x_{i} & \text { if } g_{i}(x) \geqslant 0 \\ 1 & \text { otherwise }\end{cases}
$$

This system states the Karush-Kuhn-Tucker conditions for problem (1) [9].

At the $k$ th iteration of the Newton-like method, given $x_{k}>0$, one has to solve the following linear system:

$$
W_{k} D_{k} M_{k} p=-W_{k} D_{k} g_{k}
$$


where, for $x>0$, matrices $M(x)$ and $W(x)$ are given by

$$
\begin{aligned}
& M(x)=A^{\mathrm{T}} A+D(x)^{-1} E(x) \\
& E(x)=\operatorname{diag}\left(e_{1}(x), \ldots, e_{n}(x)\right)
\end{aligned}
$$

with

$$
e_{i}(x)= \begin{cases}g_{i}(x) & \text { if } 0 \leqslant g_{i}(x)<x_{i}^{2} \text { or } g_{i}(x)^{2}>x_{i} \\ 0 & \text { otherwise }\end{cases}
$$

and

$$
W(x)=\operatorname{diag}\left(w_{1}(x), \ldots, w_{n}(x)\right), \quad w_{i}(x)=\frac{1}{d_{i}(x)+e_{i}(x)}
$$

We refer to [3] for the motivation to consider such a Newton equation. Here we just mention that this choice of $E$ and $W$ allows one to develop fast convergent methods without assuming strict complementarity at $x^{*}$.

Clearly, for $x>0$, matrices $D(x)$ and $W(x)$ are invertible and positive definite, whereas matrix $E(x)$ is positive semidefinite. Further, matrix $(W(x) D(x) M(x))^{-1}$ exists and is uniformly bounded for all strictly positive $x$, see [4, Lemma 2].

For the sake of generality, in the sequel we consider the formulation of the method in the context of inexact Newton methods [10]. Thus, the Newton equation takes the form

$$
W_{k} D_{k} M_{k} p_{k}=-W_{k} D_{k} g_{k}+r_{k}
$$

and the residual vector $r_{k}$ is required to satisfy

$$
\left\|r_{k}\right\| \leqslant \eta_{k}\left\|W_{k} D_{k} g_{k}\right\|, \quad \eta_{k} \in[0,1)
$$

The linear system (10) can be advantageously formulated as a linear system with symmetric positive-definite matrix. To this end, for any $x>0$, we let

$$
\begin{aligned}
& S(x)=W(x)^{1 / 2} D(x)^{1 / 2} \\
& Z(x)=S(x) M(x) S(x)=S(x)^{\mathrm{T}} A^{\mathrm{T}} A S(x)+W(x) E(x)
\end{aligned}
$$

and reformulate (10) as the equivalent system

$$
Z_{k} \tilde{p}_{k}=-S_{k} g_{k}+\tilde{r}_{k}
$$

with $\tilde{r}_{k}=S_{k}^{-1} r_{k}$ and $\tilde{p}_{k}=S_{k}^{-1} p_{k}$. This system has nice features. Since matrix $S(x)$ is invertible for any $x>0$, matrix $Z(x)$ is symmetric positive definite for $x>0$. Moreover it is remarkable that for $x_{k}>0$ matrix $Z_{k}$ has uniformly bounded inverse and its conditioning is not worse than that of $W_{k} D_{k} M_{k}$ [3, Lemma 2.1]. Moreover, note that from the definition of $S$ and $W$ it follows

$$
S_{k}^{2}+W_{k} E_{k}=I
$$

The residual control associated with (14) can be performed imposing

$$
\left\|\tilde{r}_{k}\right\| \leqslant \eta_{k}\left\|W_{k} D_{k} g_{k}\right\|, \quad \eta_{k} \in[0,1)
$$

Since $\left\|S_{k}\right\| \leqslant 1$, this control implies that $r_{k}=S_{k} \tilde{r}_{k}$ satisfies (11). 
After computing $\tilde{p}_{k}$ satisfying (14) and (16), the iterate $x_{k+1}$ can be formed. Specifically, positive iterates are required so that matrix $S_{k}$ is invertible. Then, $p_{k}=S_{k} \tilde{p}_{k}$ is set and the following vector $\hat{p}_{k}$ is formed:

$$
\hat{p}_{k}=\max \left\{\sigma, 1-\left\|P\left(x_{k}+p_{k}\right)-x_{k}\right\|\right\}\left(P\left(x_{k}+p_{k}\right)-x_{k}\right)
$$

where $\sigma \in(0,1)$ is close to 1 , and $P(x)=\max \{0, x\}$ is the projection of $x$ onto the positive orthant. Finally, the new iterate takes the form

$$
x_{k+1}=x_{k}+\hat{p}_{k}
$$

The purpose of this section is to investigate the solution of the Newton equation further. Clearly, the system

$$
Z_{k} \tilde{p}_{k}=-S_{k} g_{k}
$$

represents the normal equations for the least-squares problem

$$
\min _{\tilde{p} \in \mathbb{R}^{n}}\left\|B_{k} \tilde{p}+h_{k}\right\|
$$

with

$$
B_{k}=\left(\begin{array}{c}
A S_{k} \\
W_{k}^{1 / 2} E_{k}^{1 / 2}
\end{array}\right), \quad h_{k}=\left(\begin{array}{c}
A x_{k}-b \\
0
\end{array}\right)
$$

The conditioning $\kappa_{2}\left(Z_{k}\right)$ of matrix $Z_{k}$ in the 2-norm is the square of $\kappa_{2}\left(B_{k}\right)$. Clearly, if $W_{k} E_{k}=0$ then $S_{k}=I$ and $\kappa_{2}\left(B_{k}\right)=\kappa_{2}\left(A S_{k}\right)=\kappa_{2}(A)$. Otherwise, letting

$$
0<\sigma_{1} \leqslant \sigma_{2} \cdots \leqslant \sigma_{n}
$$

be the singular values of $A S_{k}$ we note that the minimum and maximum eigenvalues $\lambda_{\min }$ and $\lambda_{\max }$ of $Z_{k}$ satisfy

$$
\begin{aligned}
& \lambda_{\min }\left(Z_{k}\right) \geqslant \sigma_{1}^{2}+\min _{i}\left(w_{k} e_{k}\right)_{i} \geqslant \sigma_{1}^{2} \\
& \lambda_{\max }\left(Z_{k}\right) \leqslant \sigma_{n}^{2}+\max _{i}\left(w_{k} e_{k}\right)_{i} \leqslant \sigma_{n}^{2}+1
\end{aligned}
$$

Thus, an upper bound on the conditioning of $B_{k}$ is given by

$$
\kappa_{2}\left(B_{k}\right) \leqslant \frac{\sqrt{1+\sigma_{n}^{2}}}{\sigma_{1}} \leqslant \frac{1+\sigma_{n}}{\sigma_{1}}
$$

To avoid solving system (19), we consider the augmented system approach for the solution of the least-squares problem (20). It consists in solving the linear system

$$
\left(\begin{array}{cc}
I & A S_{k} \\
S_{k} A^{\mathrm{T}} & -W_{k} E_{k}
\end{array}\right)\left(\begin{array}{c}
\tilde{q}_{k} \\
\tilde{p}_{k}
\end{array}\right)=\left(\begin{array}{c}
-\left(A x_{k}-b\right) \\
0
\end{array}\right)
$$


To study the spectral properties of the augmented matrix, note that $W_{k} E_{k}$ is positive semidefinite and

$$
v^{\mathrm{T}} W_{k} E_{k} v \geqslant \delta v^{\mathrm{T}} v \quad \forall v \in \mathbb{R}^{n}
$$

where $1>\delta=\min _{i}\left(e_{k}\right)_{i} /\left(\left(d_{k}\right)_{i}+\left(e_{k}\right)_{i}\right)$. Clearly, the scalar $\delta$ is null whenever at least one diagonal element $\left(e_{k}\right)_{i}$ is null. The next lemma provides a bound on the conditioning of the following augmented matrix:

$$
\mathscr{H}_{\delta}=\left(\begin{array}{cc}
I & A S_{k} \\
S_{k} A^{\mathrm{T}} & -W_{k} E_{k}
\end{array}\right)
$$

Lemma 2.1

Let $0<\sigma_{1} \leqslant \sigma_{2} \cdots \leqslant \sigma_{n}$ be the singular values of $A S_{k}, \delta \in[0,1)$ be the scalar given in (25), $\mathscr{H}_{\delta}$ be the augmented matrix in (26). Then

$$
\kappa_{2}\left(\mathscr{H}_{\delta}\right) \leqslant \frac{\frac{1}{2}\left(1+\sqrt{1+4 \sigma_{n}^{2}}\right)}{\min \left\{1, \frac{1}{2}\left(\delta-1+\sqrt{(1+\delta)^{2}+4 \sigma_{1}^{2}}\right)\right\}}
$$

Proof

We order the eigenvalues of $\mathscr{H}_{\delta}$ as

$$
\mu_{-n} \leqslant \mu_{-n+1} \leqslant \cdots \leqslant \mu_{-1} \leqslant 0 \leqslant \mu_{1} \leqslant \mu_{2} \leqslant \cdots \leqslant \mu_{m}
$$

Proceeding as in $[11$, Lemmas $2.1,2.2]$ we obtain

$$
\begin{aligned}
\mu_{-n} & \geqslant-\sqrt{1+\sigma_{n}^{2}} \\
\mu_{1} & \geqslant 1 \\
\mu_{m} & \leqslant \frac{1}{2}\left(1+\sqrt{1+4 \sigma_{n}^{2}}\right)
\end{aligned}
$$

To derive an estimate for $\mu_{-1}$, let $\left(v_{1}^{\mathrm{T}}, v_{2}^{\mathrm{T}}\right)^{\mathrm{T}} \in \mathbb{R}^{m+n}$ be an eigenvector corresponding to $\mu_{-1}$. Hence, from the definition of $\mathscr{H}_{\delta}$ we have $\left(1-\mu_{-1}\right) v_{1}=-A S_{k} v_{2}$ and $v_{2}^{\mathrm{T}} S_{k} A^{\mathrm{T}} v_{1}-v_{2}^{\mathrm{T}} W_{k} E_{k} v_{2}=\mu_{-1} v_{2}^{\mathrm{T}} v_{2}$. Consequently,

$$
\left(1-\mu_{-1}\right)^{-1} v_{2}^{\mathrm{T}} S_{k} A^{\mathrm{T}} A S_{k} v_{2}+v_{2}^{\mathrm{T}} W_{k} E_{k} v_{2}=-\mu_{-1} v_{2}^{\mathrm{T}} v_{2}
$$

Since $v_{2}^{\mathrm{T}} S_{k} A^{\mathrm{T}} A S_{k} v_{2} \geqslant \sigma_{1}^{2} v_{2}^{\mathrm{T}} v_{2}$ and $v_{2}^{\mathrm{T}} W_{k} E_{k} v_{2} \geqslant \delta v_{2}^{\mathrm{T}} v_{2}$, we get $\sigma_{1}^{2}\left(1-\mu_{-1}\right)^{-1}+\delta \leqslant-\mu_{-1}$, i.e.

$$
\mu_{-1} \leqslant \frac{1}{2}\left(1-\delta-\sqrt{(1+\delta)^{2}+4 \sigma_{1}^{2}}\right)
$$

Noting that $\kappa_{2}\left(\mathscr{H}_{\delta}\right)=\max \left\{\left|\mu_{-n}\right|, \mu_{m}\right\} / \min \left\{\left|\mu_{-1}\right|, \mu_{1}\right\}$, the thesis follows from (28)-(31).

Taking into account (25), the bound (31) is sharper than that provided in [11]. Also, our results are a generalization of those given in [12]. 
If $\delta=0$, noting that $\sqrt{1+4 \sigma_{n}^{2}}<1+2 \sigma_{n}$, Lemma 2.1 yields

$$
\kappa_{2}\left(\mathscr{H}_{0}\right) \leqslant \frac{1+\sigma_{n}}{\min \left\{1, \frac{1}{2}\left(-1+\sqrt{1+4 \sigma_{1}^{2}}\right)\right\}}
$$

Now, suppose $\sigma_{1}$ is significantly smaller than 1 . Since $\frac{1}{2}\left(-1+\sqrt{1+4 \sigma_{1}^{2}}\right) \simeq \sigma_{1}^{2}$, it follows that $\kappa_{2}\left(\mathscr{H}_{0}\right)$ may be much greater than $\kappa_{2}\left(B_{k}\right)$ (see (23)). On the other hand, if $\delta \neq 0$, the augmented system can be viewed as a regularized system and the regularization can improve the conditioning of the system. To show this fact we proceed as in (32) and noting that $\sqrt{(1+\delta)^{2}+4 \sigma_{1}^{2}}>1+\delta$ we get

$$
\kappa_{2}\left(\mathscr{H}_{\delta}\right) \leqslant \frac{1+\sigma_{n}}{\min \left\{1, \frac{1}{2}\left(\delta-1+\sqrt{(1+\delta)^{2}+4 \sigma_{1}^{2}}\right)\right\}} \leqslant \frac{1+\sigma_{n}}{\delta}
$$

Therefore, when $\sigma_{1}$ is small and $\delta>\sigma_{1}$, the condition number of $\kappa_{2}\left(\mathscr{H}_{\delta}\right)$ may be considerably smaller than $\kappa_{2}\left(\mathscr{H}_{0}\right)$.

So far we have assumed that $\sigma_{1}$ is small. If this is not the case, the regularization does not deteriorate $\kappa_{2}\left(\mathscr{H}_{\delta}\right)$ with respect to $\kappa_{2}\left(\mathscr{H}_{0}\right)$, e.g. if $\sigma_{1} \geqslant 1$ and $\delta \leqslant \frac{1}{2}$ we have $(1+\delta)^{2}+4 \sigma_{1}^{2} \geqslant(2+\delta)^{2}$, and the bound on $\kappa_{2}\left(\mathscr{H}_{\delta}\right)$ becomes

$$
\kappa_{2}\left(\mathscr{H}_{\delta}\right) \leqslant \frac{1+\sigma_{n}}{\frac{1}{2}+\delta}
$$

This discussion suggests that ensuring $\delta>0$ is a good strategy in order to overcome the potential ill-conditioning of the augmented system. In particular, the regularization is useful when $\sigma_{1}$ is much smaller than 1 and the scalar $\delta$ in (25) is such that $\delta>\sigma_{1}$.

\section{THE REGULARIZED NEWTON-LIKE METHOD}

Here we propose a modification of the Newton-like method given in [3] that gives rise to a regularized augmented system. In this manner, the potential ill-conditioning of the augmented system is avoided.

Since the scalar $\delta$ is null if at least one element of matrix $E_{k}$ given in (8) is null, to regularize system $(24)$ we need to modify the $(2,2)$ block of the augmented matrix. To this end, we replace (10) with the Newton equation

$$
W_{k} D_{k} N_{k} p_{k}=-W_{k} D_{k} g_{k}+r_{k}
$$

where

$$
\begin{aligned}
& N_{k}=A^{\mathrm{T}} A+D_{k}^{-1} E_{k}+\Delta_{k} \\
& \Delta_{k}=\operatorname{diag}\left(\delta_{k, 1}, \delta_{k, 2}, \ldots, \delta_{k, n}\right), \quad \delta_{k, i} \in[0,1), \quad i=1, \ldots, n
\end{aligned}
$$

and $r_{k}$ satisfies (11). From Lemma 2 of [4] it can be easily derived that matrix $W_{k} D_{k} N_{k}$ is invertible for any $x_{k}>0$ and there exists a constant $\bar{C}$ independent of $k$ such that

$$
\left\|\left(W_{k} D_{k} N_{k}\right)^{-1}\right\|<\bar{C}
$$


Proceeding as to obtain (14), (34) can be reformulated as the following symmetric positive-definite system:

$$
S_{k} N_{k} S_{k} \tilde{p}_{k}=-S_{k} g_{k}+\tilde{r}_{k}
$$

with $\tilde{p}_{k}=S_{k}^{-1} p_{k}$ and $\tilde{r}_{k}$ satisfying (16). form

If $\delta_{k, i}$ is strictly positive whenever $\left(e_{k}\right)_{i}=0$, the augmented system is regularized and takes the

$$
\begin{aligned}
\left(\begin{array}{cc}
I & A S_{k} \\
S_{k} A^{\mathrm{T}} & -C_{k}
\end{array}\right)\left(\begin{array}{l}
\tilde{q}_{k} \\
\tilde{p}_{k}
\end{array}\right) & =\left(\begin{array}{c}
-\left(A x_{k}-b\right) \\
0
\end{array}\right) \\
C_{k} & =W_{k} E_{k}+\Delta_{k} S_{k}^{2}
\end{aligned}
$$

The Newton-like method proposed can be globalized using a simple strategy analogous to the one in [3]. Following such strategy, the new iterate $x_{k+1}$ is required to satisfy

$$
\frac{\psi_{k}\left(x_{k+1}-x_{k}\right)}{\psi_{k}\left(p_{k}^{C}\right)} \geqslant \beta, \quad \beta \in(0,1)
$$

where, given $p \in \mathbb{R}^{n}, \psi_{k}(p)$ is the following quadratic function:

$$
\psi_{k}(p)=\frac{1}{2} p^{\mathrm{T}} N_{k} p+p^{\mathrm{T}} g_{k}
$$

and the step $p_{k}^{C}$ is a constrained scaled Cauchy step that approximates the solution to the problem

$$
\operatorname{argmin}\left\{\psi_{k}(p): p=-c_{k} D_{k} g_{k}, c_{k}>0, x_{k}+p>0\right\}
$$

In practice, $p_{k}^{C}$ is given by

$$
p_{k}^{C}=-c_{k} D_{k} g_{k}
$$

with

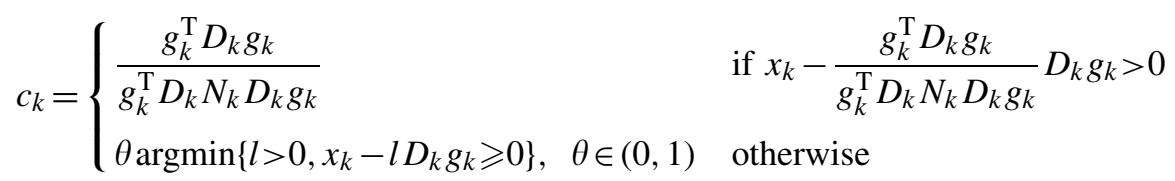

In particular, letting $p_{k}$ be a step satisfying (34) and $\hat{p}_{k}$ be the step defined in (17), the new iterate has the form

$$
x_{k+1}=x_{k}+t p_{k}^{C}+(1-t) \hat{p}_{k}
$$

If the point $x_{k+1}=x_{k}+\hat{p}_{k}$ satisfies (41), $t$ is simply taken equal to zero, otherwise a scalar $t \in(0,1]$ is computed in order to satisfy (41). The computation of such $t$ can be performed inexpensively as shown in [3].

The global and local convergence properties of the regularized Newton-like method are proved in the next theorem. To maintain fast convergence, eventually it is necessary to control the forcing term $\eta_{k}$ in (16) and the entries of $\Delta_{k}$. 


\section{Theorem 3.1}

Let $x_{0}$ be an arbitrary strictly positive initial point.

(i) The sequence $\left\{x_{k}\right\}$ generated by the regularized Newton-like method converges to $x^{*}$.

(ii) If $\left\|\Delta_{k}\right\| \leqslant \Lambda_{1}\left\|W_{k} D_{k} g_{k}\right\|$ and $\eta_{k} \leqslant \Lambda_{2}\left\|W_{k} D_{k} g_{k}\right\|$, for some positive $\Lambda_{1}, \Lambda_{2}$, and $k$ sufficiently large, then the sequence $\left\{x_{k}\right\}$ converges $q$-quadratically toward $x^{*}$.

Proof

(i) Note that

$$
q\left(x_{k}\right)-q\left(x_{k}+p\right)=-\psi_{k}(p)+\frac{1}{2} p^{\mathrm{T}}\left(\Delta_{k}+D_{k}^{-1} E_{k}\right) p>-\psi_{k}(p)
$$

for any $p \in \mathbb{R}^{n}$. Then, proceeding as in Theorem 2.2 in [3] we easily get the thesis.

(ii) In order to prove quadratic rate of convergence, we estimate the norm of the vector $\left(x_{k}+p_{k}-\right.$ $x^{*}$ ) where $p_{k}$ solves (34) and $r_{k}$ satisfies (11). Subtracting the trivial equality $W_{k} D_{k} N_{k}\left(x^{*}-x^{*}\right)=$ $-W_{k} D\left(x^{*}\right) g\left(x^{*}\right)$ from (34) with a little algebra we get

$$
W_{k} D_{k} N_{k}\left(x_{k}+p_{k}-x^{*}\right)=W_{k} \rho_{k}
$$

where

$$
\rho_{k}=D_{k} \Delta_{k}\left(x_{k}-x^{*}\right)+W_{k}^{-1} r_{k}-\left(D_{k}-D\left(x^{*}\right)\right) g\left(x^{*}\right)+E_{k}\left(x_{k}-x^{*}\right)
$$

Letting $\tilde{\rho}_{k}$ be the vector such that

$$
\tilde{\rho}_{k}=W_{k}\left(-\left(D_{k}-D\left(x^{*}\right)\right) g\left(x^{*}\right)+E_{k}\left(x_{k}-x^{*}\right)\right)
$$

and using (11) we get

$$
\begin{aligned}
\left\|W_{k} \rho_{k}\right\| & \leqslant\left\|W_{k} D_{k}\right\|\left\|\Delta_{k}\right\|\left\|x_{k}-x^{*}\right\|+\left\|r_{k}\right\|+\left\|\tilde{\rho}_{k}\right\| \\
& \leqslant\left\|\Delta_{k}\right\|\left\|x_{k}-x^{*}\right\|+\eta_{k}\left\|W_{k} D_{k} g_{k}\right\|+\left\|\tilde{\rho}_{k}\right\|
\end{aligned}
$$

Then, from (37), (44) and (45) it follows

$$
\left\|x_{k}+p_{k}-x^{*}\right\| \leqslant \bar{C}\left(\left\|\Delta_{k}\right\|\left\|x_{k}-x^{*}\right\|+\eta_{k}\left\|W_{k} D_{k} g_{k}\right\|+\left\|\tilde{\rho}_{k}\right\|\right)
$$

Moreover, from the proof of Theorem 4 in [4] it can be derived that there exist constants $C_{1}>0$ and $r_{1}>0$ such that

$$
\left\|\tilde{\rho}_{k}\right\| \leqslant C_{1}\left\|x_{k}-x^{*}\right\|^{2}
$$

whenever $\left\|x_{k}-x^{*}\right\| \leqslant r_{1}$. Finally, from the proof of Lemma 3.2 of [3] it follows that there are $C_{2}>0$ and $r_{2}>0$ such that

$$
\left\|W_{k} D_{k} g_{k}\right\| \leqslant C_{2}\left\|x_{k}-x^{*}\right\|
$$

whenever $\left\|x_{k}-x^{*}\right\| \leqslant r_{2}$. Then, (47) and (48) along with (46) yield

$$
\left\|x_{k}+p_{k}-x^{*}\right\| \leqslant \bar{C}\left(\left\|\Delta_{k}\right\|+C_{2} \eta_{k}+C_{1}\left\|x^{*}-x_{k}\right\|\right)\left\|x^{*}-x_{k}\right\|
$$

whenever $\left\|x_{k}-x^{*}\right\| \leqslant \min \left(r_{1}, r_{2}\right)$. Therefore, under the assumptions $\left\|\Delta_{k}\right\| \leqslant \Lambda_{1}\left\|W_{k} D_{k} g_{k}\right\|$ and $\eta_{k} \leqslant \Lambda_{2}\left\|W_{k} D_{k} g_{k}\right\|$ for sufficiently large $k$, there exist $C>0$ and $r>0$ such that

$$
\left\|x_{k}+p_{k}-x^{*}\right\| \leqslant C\left\|x^{*}-x_{k}\right\|^{2}
$$


whenever $\left\|x_{k}-x^{*}\right\| \leqslant r$. With this result at hand, slight modifications in the proofs of Lemmas 3.2, 3.3 and Theorem 3.1 of [3] yield the thesis.

We underline that Theorem 3.1 holds even if $x^{*}$ is degenerate and the convergence properties of the method in [3] are not degraded.

\section{ITERATIVE LINEAR ALGEBRA}

In this section we focus on the solution of the Newton equation (34) via the augmented system (39) employing an iterative method.

It is interesting to characterize the entries of matrix $S(x)$ given in (12). When the sequence $\left\{x_{k}\right\}$ generated by our method converges to the solution $x^{*}$, the entries of $S_{k}$ corresponding to the active nondegenerate and possibly degenerate components of $x^{*}$ tend to zero. Therefore there is a splitting of matrix $S_{k}$ in two diagonal blocks $\left(S_{k}\right)_{1}$ and $\left(S_{k}\right)_{2}$ such that $\lim _{k \rightarrow \infty}\left(S_{k}\right)_{1}=I$, $\lim _{k \rightarrow \infty}\left(S_{k}\right)_{2}=0$.

More generally, given a small positive threshold $\tau \in(0,1)$, at each iteration we let

$$
\mathscr{L}_{k}=\left\{i \in\{1,2, \ldots, n\}, \text { s.t. }\left(s_{k}\right)_{i}^{2} \geqslant 1-\tau\right\}, \quad n_{1}=\operatorname{card}\left(\mathscr{L}_{k}\right)
$$

where $\operatorname{card}\left(\mathscr{L}_{k}\right)$ is the cardinality of the set $\mathscr{L}_{k}$. Then, for simplicity we omit permutations and assume that

$$
\begin{gathered}
S_{k}=\left(\begin{array}{cc}
\left(S_{k}\right)_{1} & 0 \\
0 & \left(S_{k}\right)_{2}
\end{array}\right) \\
\left(S_{k}\right)_{1}=\operatorname{diag}_{i \in \mathscr{L}_{k}}\left(\left(s_{k}\right)_{i}\right) \in \mathbb{R}^{n_{1} \times n_{1}} \\
\left(S_{k}\right)_{2}=\operatorname{diag}_{i \notin \mathscr{L}_{k}}\left(\left(s_{k}\right)_{i}\right) \in \mathbb{R}^{\left(n-n_{1}\right) \times\left(n-n_{1}\right)}
\end{gathered}
$$

Analogously for any diagonal matrix $G \in \mathbb{R}^{n \times n}$ let $(G)_{1} \in \mathbb{R}^{n_{1} \times n_{1}}$ be the submatrix formed by the first $n_{1}$ rows and $n_{1}$ columns and $(G)_{2} \in \mathbb{R}^{\left(n-n_{1}\right) \times\left(n-n_{1}\right)}$ be the submatrix formed by the remaining rows and columns. Finally, we consider the partitioning $A=\left(A_{1}, A_{2}\right), A_{1} \in \mathbb{R}^{m \times n_{1}}, A_{2} \in \mathbb{R}^{m \times\left(n-n_{1}\right)}$.

Assume the set $\mathscr{L}_{k}$ is nonempty. Consequently, the augmented system (39) takes the form

$$
\left(\begin{array}{ccc}
I & A_{1}\left(S_{k}\right)_{1} & A_{2}\left(S_{k}\right)_{2} \\
\left(S_{k}\right)_{1} A_{1}^{\mathrm{T}} & -\left(C_{k}\right)_{1} & 0 \\
\left(S_{k}\right)_{2} A_{2}^{\mathrm{T}} & 0 & -\left(C_{k}\right)_{2}
\end{array}\right)\left(\begin{array}{c}
\tilde{q}_{k} \\
\left(\tilde{p}_{k}\right)_{1} \\
\left(\tilde{p}_{k}\right)_{2}
\end{array}\right)=\left(\begin{array}{c}
-\left(A x_{k}-b\right) \\
0 \\
0
\end{array}\right)
$$

and eliminating $\left(\tilde{p}_{k}\right)_{2}$ from the first equation we get

$$
\underbrace{\left(\begin{array}{cc}
I+Q_{k} & A_{1}\left(S_{k}\right)_{1} \\
\left(S_{k}\right)_{1} A_{1}^{\mathrm{T}} & -\left(C_{k}\right)_{1}
\end{array}\right)}_{\mathscr{A}_{k}}\left(\begin{array}{c}
\tilde{q}_{k} \\
\left(\tilde{p}_{k}\right)_{1}
\end{array}\right)=\left(\begin{array}{c}
-\left(A x_{k}-b\right) \\
0
\end{array}\right)
$$


where $Q_{k}=A_{2}\left(S_{k} C_{k}^{-1} S_{k}\right)_{2} A_{2}^{\mathrm{T}}$. We note that

$$
\mathscr{A}_{k}=\left(\begin{array}{cc}
I & A_{1}\left(S_{k}\right)_{1} \\
\left(S_{k}\right)_{1} A_{1}^{\mathrm{T}} & -\left(\Delta_{k} S_{k}^{2}\right)_{1}
\end{array}\right)+\left(\begin{array}{cc}
Q_{k} & 0 \\
0 & -\left(W_{k} E_{k}\right)_{1}
\end{array}\right)
$$

and precondition (55) with the matrix

$$
\mathscr{P}_{k}=\left(\begin{array}{cc}
I & A_{1}\left(S_{k}\right)_{1} \\
\left(S_{k}\right)_{1} A_{1}^{\mathrm{T}} & -\left(\Delta_{k} S_{k}^{2}\right)_{1}
\end{array}\right)
$$

The preconditioner $\mathscr{P}_{k}$ has the following features. As $\left(w_{k}\right)_{i}\left(e_{k}\right)_{i}+\left(s_{k}\right)_{i}^{2}=1$ for $i=1, \ldots, n$, by definition (51) we have $\left\|\left(W_{k} E_{k}\right)_{1}\right\| \leqslant \tau,\left\|\left(C_{k}\right)_{2}^{-1}\right\| \leqslant 1 / \tau,\left\|Q_{k}\right\| \leqslant(1-\tau)\left\|A_{2}\right\|^{2} / \tau$; further, when $\left\{x_{k}\right\}$ approaches the solution $x^{*}$ (hence $\left(S_{k}\right)_{1} \rightarrow I$ and $\left.\left(S_{k}\right)_{2} \rightarrow 0\right)$ eventually both $Q_{k}$ and $\left(W_{k} E_{k}\right)_{1}$ tend to zero, i.e. $\left\|\mathscr{P}_{k}-\mathscr{A}_{k}\right\|$ tends to zero.

Let us observe that the factorization of $\mathscr{P}_{k}$ can be accomplished based on the identity

$$
\mathscr{P}_{k}=\left(\begin{array}{cc}
I & 0 \\
0 & \left(S_{k}\right)_{1}
\end{array}\right) \underbrace{\left(\begin{array}{cc}
I & A_{1} \\
A_{1}^{\mathrm{T}} & -\left(\Delta_{k}\right)_{1}
\end{array}\right)}_{\Pi_{k}}\left(\begin{array}{cc}
I & 0 \\
0 & \left(S_{k}\right)_{1}
\end{array}\right)
$$

and factorizing $\Pi_{k}$. If the set $\mathscr{L}_{k}$ and matrix $\Delta_{k}$ remain unchanged for a few iterations, the factorization of the matrix $\Pi_{k}$ does not have to be updated. In fact, eventually $\mathscr{L}_{k}$ is expected to settle down as it contains the indices of all the inactive components of $x^{*}$ and the indices of the degenerate components $i$ such that $\left(s_{k}\right)_{i}$ tends to one.

The augmented system (55) can be solved by iterative methods for indefinite systems, e.g. BiCGSTAB [13], GMRES [14], QMR [15]. The speed of convergence of these methods depends on the spectral properties of the preconditioned matrix $\mathscr{P}_{k}^{-1} \mathscr{A}_{k}$, which are provided in the following theorem.

\section{Theorem 4.1}

Let $\mathscr{A}_{k}$ and $\mathscr{P}_{k}$ be the matrices given in (55) and (57). Then at least $m-n+n_{1}$ eigenvalues of $\mathscr{P}_{k}^{-1} \mathscr{A}_{k}$ are unit and the other eigenvalues are positive and of the form

$$
\lambda=1+\mu, \quad \mu=\frac{u^{\mathrm{T}} Q_{k} u+v^{\mathrm{T}}\left(W_{k} E_{k}\right)_{1} v}{u^{\mathrm{T}} u+v^{\mathrm{T}}\left(\Delta_{k} S_{k}^{2}\right)_{1} v}
$$

where $\left(u^{\mathrm{T}}, v^{\mathrm{T}}\right)^{\mathrm{T}}$ is an eigenvector associated with $\lambda$.

\section{Proof}

The eigenvalues and eigenvectors of matrix $\mathscr{P}_{k}^{-1} \mathscr{A}_{k}$ satisfy

$$
\left(\begin{array}{cc}
I+Q_{k} & A_{1}\left(S_{k}\right)_{1} \\
\left(S_{k}\right)_{1} A_{1}^{\mathrm{T}} & -\left(C_{k}\right)_{1}
\end{array}\right)\left(\begin{array}{l}
u \\
v
\end{array}\right)=\lambda\left(\begin{array}{cc}
I & A_{1}\left(S_{k}\right)_{1} \\
\left(S_{k}\right)_{1} A_{1}^{\mathrm{T}} & -\left(\Delta_{k} S_{k}^{2}\right)_{1}
\end{array}\right)\left(\begin{array}{l}
u \\
v
\end{array}\right)
$$

If $\lambda=1$ we get

$$
\begin{aligned}
\left(I+Q_{k}\right) u & =u \\
\left(C_{k}\right)_{1} v & =\left(\Delta_{k} S_{k}^{2}\right)_{1} v
\end{aligned}
$$


that is $u$ belongs to the null space of $Q_{k}$ and $v$ belongs to the null space of $\left(W_{k} E_{k}\right)_{1}$. As $\operatorname{rank}\left(Q_{k}\right)=n-n_{1}$, it follows that there are at least $m-\left(n-n_{1}\right)$ unit eigenvalues. If $\lambda \neq 1$, denoting $\lambda=1+\mu$ we have

$$
\begin{aligned}
u^{\mathrm{T}} Q_{k} u & =\mu u^{\mathrm{T}} u+\mu u^{\mathrm{T}} A_{1}\left(S_{k}\right)_{1} v \\
v^{\mathrm{T}}\left(W_{k} E_{k}\right)_{1} v & =-\mu v^{\mathrm{T}}\left(S_{k}\right)_{1} A_{1}^{\mathrm{T}} u+\mu v^{\mathrm{T}}\left(\Delta_{k} S_{k}^{2}\right)_{1} v
\end{aligned}
$$

Then, adding these two equations we obtain (59). Since $Q_{k},\left(W_{k} E_{k}\right)_{1}$ and $\Delta_{k} S_{k}^{2}$ are positive semidefinite we conclude that $\mu$ is positive.

Clearly, if $\mu$ is small it means that the eigenvalues of $\mathscr{P}_{k}^{-1} \mathscr{A}_{k}$ are clustered around one and fast convergence of Krylov methods can be expected. This is the case when $x_{k}$ is close to the solution. On the other hand, when $x_{k}$ is still far away from $x^{*}$, the following bounds for $\mu$ can be derived.

Corollary 4.1

Let $\mathscr{A}_{k}$ and $\mathscr{P}_{k}$ be the matrices given in (56) and (57), $\tau$ be the scalar in (51), $\mathscr{L}_{k}$ be the set given in $(51), \overline{\mathscr{L}}_{k}=\left\{i \in \mathscr{L}_{k}:\left(s_{k}\right)_{i}^{2} \neq 1\right\}$.

If the elements $\delta_{k, i}$ in (36) are such that $\delta_{k, i}=\delta>0$ for $i \in \mathscr{L}_{k}$, and $\delta_{k, i}=0$ for $i \notin \mathscr{L}_{k}$, then the eigenvalues of $\mathscr{P}_{k}^{-1} \mathscr{A}_{k}$ have the form $\lambda=1+\mu$ and

$$
\mu \leqslant \frac{\left\|A_{2}\left(S_{k}\right)_{2}\right\|^{2}}{\tau}+\frac{\tau}{\delta(1-\tau)}
$$

If the elements $\delta_{k, i}$ in (36) are such that

$$
\delta_{k, i}= \begin{cases}\left(w_{k}\right)_{i}\left(e_{k}\right)_{i} & \text { if } i \in \overline{\mathscr{L}}_{k} \\ \left\|W_{k} D_{k} g_{k}\right\| & \text { if } i \in \mathscr{L}_{k} \backslash \overline{\mathscr{L}}_{k} \\ 0 & \text { if } i \notin \mathscr{L}_{k}\end{cases}
$$

then the eigenvalues of $\mathscr{P}_{k}^{-1} \mathscr{A}_{k}$ have the form $\lambda=1+\mu$ and

$$
\mu \leqslant \frac{\left\|A_{2}\left(S_{k}\right)_{2}\right\|^{2}}{\tau}+\frac{1}{1-\tau}
$$

Proof

Consider (59) and suppose $u$ and $v$ are not null. Then we have

$$
\mu \leqslant \frac{u^{\mathrm{T}} Q_{k} u}{u^{\mathrm{T}} u}+\frac{v^{\mathrm{T}}\left(W_{k} E_{k}\right)_{1} v}{v^{\mathrm{T}}\left(\Delta_{k} S_{k}^{2}\right)_{1} v}
$$

Also observe that (51) implies

$$
\min _{i \in \mathscr{L}_{k}}\left(s_{k}\right)_{i}^{2} \geqslant 1-\tau, \quad\left\|\left(W_{k} E_{k}\right)_{1}\right\| \leqslant \tau, \quad\left\|\left(C_{k}\right)_{2}^{-1}\right\| \leqslant \frac{1}{\tau}
$$

Then, when $\delta_{k, i}=\delta>0$ for $i \in \mathscr{L}_{k}$, we obtain

$$
\mu \leqslant\left\|\left(C_{k}\right)_{2}^{-1}\right\|\left\|A_{2}\left(S_{k}\right)_{2}\right\|^{2}+\frac{\tau}{\delta(1-\tau)}
$$

which yields (61). 
Consider (62). For $i \in \mathscr{L}_{k} \backslash \bar{L}_{k}$, we have $\left(w_{k}\right)_{i}\left(e_{k}\right)_{i}=0$. Then, we get

$$
\begin{aligned}
& \mu \leqslant\left\|\left(C_{k}\right)_{2}^{-1}\right\|\left\|A_{2}\left(S_{k}\right)_{2}\right\|^{2}+\frac{\sum_{i \in \mathscr{L}_{k}}\left(w_{k}\right)_{i}\left(e_{k}\right)_{i} v_{i}^{2}}{\sum_{i \in \mathscr{L}_{k}} \delta_{k, i}\left(s_{k}\right)_{i}^{2} v_{i}^{2}} \\
& =\left\|\left(C_{k}\right)_{2}^{-1}\right\|\left\|A_{2}\left(S_{k}\right)_{2}\right\|^{2}+\frac{\sum_{i \in \overline{\mathscr{L}}_{k}} \delta_{k, i} v_{i}^{2}}{\sum_{i \in \overline{\mathscr{L}}_{k}} \delta_{k, i}\left(s_{k}\right)_{i}^{2} v_{i}^{2}+\sum_{i \in \mathscr{L}_{k} \backslash \overline{\mathscr{L}}_{k}} \delta_{k, i}\left(s_{k}\right)_{i}^{2} v_{i}^{2}} \\
& \leqslant \frac{\left\|A_{2}\left(S_{k}\right)_{2}\right\|^{2}}{\tau}+\frac{\sum_{i \in \overline{\mathscr{L}}_{k}} \delta_{k, i} v_{i}^{2}}{\sum_{i \in \overline{\mathscr{L}}_{k}}\left(s_{k}\right)_{i}^{2} \delta_{k, i} v_{i}^{2}} \\
& \leqslant \frac{\left\|A_{2}\left(S_{k}\right)_{2}\right\|^{2}}{\tau}+\frac{1}{\min _{i \in \overline{\mathscr{L}}_{k}}\left(s_{k}\right)_{i}^{2}}
\end{aligned}
$$

Then (63) trivially follows from (51).

Finally, if either $u$ or $v$ is null the bound (59) consists in one of the two terms and the thesis still holds.

The previous result shows that the choice of the regularization parameters $\delta_{k, i}=\delta>0$ for $i \in \mathscr{L}_{k}$ does not provide good properties of the spectrum of $\mathscr{P}_{k}^{-1} \mathscr{A}_{k}$ whenever $x_{k}$ is far from $x^{*}$. In fact, to minimize the second term in (61), we should fix $\tau=O(\delta)$ but the scalar $\left\|A_{2}\left(S_{k}\right)_{2}\right\|^{2} / \tau$ may be large as $\delta$ is supposed to be small. On the other hand, letting $\delta_{k, i}$ as in (62) we have a better distribution of the eigenvalues of $\mathscr{P}_{k}^{-1} \mathscr{A}_{k}$. Note that for any regularization used it is essential to keep the term $\left\|A_{2}\left(S_{k}\right)_{2}\right\|^{2} / \tau$ as small as possible. Hence we advise scaling matrix $A$ at the beginning of the solution process to guarantee that the norm $\|A\|$ is small.

We note that the choice of the regularization parameters given in (62) provides fast convergence in most cases. In particular, if $x^{*}$ has no degenerate components, then $\left\|\Delta_{k}\right\| \leqslant \max \left\{\max _{i \in \overline{\mathscr{L}}_{k}}\left(w_{k}\right)_{i}\left(e_{k}\right)_{i}\right.$, $\left.\left\|W_{k} D_{k} g_{k}\right\|\right\}=O\left(\left\|W_{k} D_{k} g_{k}\right\|\right)$. This is due to the fact that, for inactive components,

$$
\frac{\left(w_{k}\right)_{i}\left(e_{k}\right)_{i}}{\left\|W_{k} D_{k} g_{k}\right\|} \leqslant \frac{\left(e_{k}\right)_{i}}{\left(d_{k}\right)_{i}+\left(e_{k}\right)_{i}} \frac{\left(d_{k}\right)_{i}+\left(e_{k}\right)_{i}}{\left(d_{k}\right)_{i}\left(g_{k}\right)_{i}}=\frac{1}{\left(d_{k}\right)_{i}}
$$

Thus, according to Theorem 3.1, quadratic convergence can be achieved. The same result holds if there are degenerate components either when their indices do not belong to $\mathscr{L}_{k}$ eventually or when, for infinitely many times, $\left\|\Delta_{k}\right\| \neq\left(w_{k}\right)_{i}\left(e_{k}\right)_{i}$ where $i$ corresponds to a degenerate component. In the unlikely situation where there exists at least one component degenerate such that $\left(e_{k}\right)_{i}=g_{i}(x)$ with $g_{i}(x) \in\left[0, x_{i}^{2}\right)$ and $\left\|\Delta_{k}\right\|=\left(w_{k}\right)_{i}\left(e_{k}\right)_{i}$ infinitely many times, the quadratic convergence is not guaranteed.

Our regularized augmented system equation (55) can be solved by the PPCG method developed in $[8,16]$. PPCG provides the vector $\tilde{q}_{k}$, while the vector $\tilde{p}_{k}$ is computed by $\tilde{p}_{k}=\left(C_{k}\right)^{-1}\left(S_{k}\right) A^{\mathrm{T}} \tilde{q}_{k}$. Solving (55) with preconditioner $\mathscr{P}_{k}$ by PPCG is equivalent to applying preconditioned conjugategradient (PCG) to the system

$$
\underbrace{\left(I+Q_{k}+A_{1}\left(S_{k} C_{k}^{-1} S_{k}\right){ }_{1} A_{1}^{\mathrm{T}}\right)}_{\mathscr{F}_{k}} \tilde{q}_{k}=-\left(A x_{k}-b\right)
$$


using a preconditioner of the form

$$
\mathscr{G}_{k}=I+A_{1}\left(\Delta_{k}\right)_{1}^{-1} A_{1}^{\mathrm{T}}
$$

see [17]. Thus we are interested in the distribution of the eigenvalues for matrix $\mathscr{G}_{k}^{-1} \mathscr{F}_{k}$.

Theorem 4.2

Let $\mathscr{F}_{k}$ and $\mathscr{G}_{k}$ be the matrices given in (65) and (66), $\mathscr{L}_{k}$ be the set given in (51), $\overline{\mathscr{L}}_{k}=\left\{i \in \mathscr{L}_{k}\right.$ : $\left.\left(s_{k}\right)_{i}^{2} \neq 1\right\}$. If $\delta_{k, i}$ are given by (62), then the eigenvalues of $\mathscr{G}_{k}^{-1} \mathscr{F}_{k}$ satisfy

$$
1-\frac{1}{2-\tau} \leqslant \lambda \leqslant 1+\frac{\left\|A_{2}\left(S_{k}\right)_{2}\right\|^{2}}{\tau}
$$

Proof

Let $\lambda$ and $u \in \mathbb{R}^{m}$ be the eigenvalues and eigenvectors of $\mathscr{G}_{k}^{-1} \mathscr{F}_{k}$. Then $\lambda$ and $u$ satisfy

$$
\lambda=\frac{u^{\mathrm{T}}\left(I+Q_{k}+A_{1}\left(S_{k} C_{k}^{-1} S_{k}\right)_{1} A_{1}^{\mathrm{T}}\right) u}{u^{\mathrm{T}}\left(I+A_{1}\left(\Delta_{k}\right)_{1}^{-1} A_{1}^{\mathrm{T}}\right) u}
$$

Then, noting that for any $z>0$ and positive $a$ and $b$ such that $b>a$ we have $(z+a) /(z+b)>a / b$, it follows

$$
\begin{aligned}
\lambda> & \frac{u^{\mathrm{T}} A_{1}\left(S_{k} C_{k}^{-1} S_{k}\right)_{1} A_{1}^{\mathrm{T}} u}{u^{\mathrm{T}} A_{1}\left(\Delta_{k}\right)_{1}^{-1} A_{1}^{\mathrm{T}} u} \\
& =\frac{\left(\left(\Delta_{k}\right)_{1}^{-1 / 2} A_{1}^{\mathrm{T}} u\right)^{\mathrm{T}}\left(S_{k}\right)_{1}\left(\left(W_{k} E_{k}\right)_{1}\left(\Delta_{k}\right)^{-1}+\left(S_{k}^{2}\right)_{1}\right)^{-1}\left(S_{k}\right)_{1}\left(\left(\Delta_{k}\right)_{1}^{-1 / 2} A_{1}^{\mathrm{T}} u\right)}{\left\|\left(\Delta_{k}\right)_{1}^{-1 / 2} A_{1}^{\mathrm{T}} u\right\|^{2}}
\end{aligned}
$$

Letting $z=\left(\Delta_{k}\right)_{1}^{-1 / 2} A_{1}^{\mathrm{T}} u$ we obtain

$$
\lambda \geqslant \frac{\sum_{i \in \mathscr{L}_{k}} \frac{\delta_{k, i}\left(s_{k}\right)_{i}^{2} z_{i}^{2}}{\left(w_{k}\right)_{i}\left(e_{k}\right)_{i}+\delta_{k, i}\left(s_{k}\right)_{i}^{2}}}{\sum_{i \in \mathscr{L}_{k}} z_{i}^{2}}=\frac{\sum_{i \in \overline{\mathscr{L}}_{k}} \frac{\delta_{k, i}\left(s_{k}\right)_{i}^{2} z_{i}^{2}}{\left(w_{k}\right)_{i}\left(e_{k}\right)_{i}+\delta_{k, i}\left(s_{k}\right)_{i}^{2}}+\sum_{i \in \mathscr{L}_{k} \backslash \overline{\mathscr{L}}_{k}} z_{i}^{2}}{\sum_{i \in \mathscr{L}_{k}} z_{i}^{2}}
$$

This implies

$$
\begin{aligned}
\lambda \geqslant & \frac{\min _{i \in \overline{\mathscr{L}}_{k}} \frac{\delta_{k, i}\left(s_{k}\right)_{i}^{2}}{\left(w_{k}\right)_{i}\left(e_{k}\right)_{i}+\delta_{k, i}\left(s_{k}\right)_{i}^{2}} \sum_{i \in \overline{\mathscr{L}}_{k}} z_{i}^{2}+\sum_{i \in \mathscr{L}_{k} \backslash \overline{\mathscr{L}}_{k}} z_{i}^{2}}{\sum_{i \in \mathscr{L}_{k}} z_{i}^{2}} \\
& \geqslant \min _{i \in \overline{\mathscr{L}}_{k}} \frac{\delta_{k, i}\left(s_{k}\right)_{i}^{2}}{\left(w_{k}\right)_{i}\left(e_{k}\right)_{i}+\delta_{k, i}\left(s_{k}\right)_{i}^{2}}
\end{aligned}
$$


and for $i \in \overline{\mathscr{L}}_{k}$

$$
\begin{aligned}
\frac{\delta_{k, i}\left(s_{k}\right)_{i}^{2}}{\left(w_{k}\right)_{i}\left(e_{k}\right)_{i}+\delta_{k, i}\left(s_{k}\right)_{i}^{2}} & =1-\frac{\left(w_{k}\right)_{i}\left(e_{k}\right)_{i}}{\left(w_{k}\right)_{i}\left(e_{k}\right)_{i}+\delta_{k, i}\left(s_{k}\right)_{i}^{2}} \\
& =1-\frac{1}{1+\left(s_{k}\right)_{i}^{2}} \\
& \geqslant 1-\frac{1}{2-\tau}
\end{aligned}
$$

This yields the lower bound in (67).

Concerning the upper bound on $\lambda$, first observe that for any vector $v \in \mathbb{R}^{n_{1}}$, we have $v^{\mathrm{T}}\left(S_{k} C_{k}^{-1} S_{k}\right)_{1} v \leqslant v^{\mathrm{T}}\left(\Delta_{k}\right)_{1}^{-1} v$. Hence,

$$
\begin{aligned}
\lambda & =\frac{u^{\mathrm{T}}\left(I+Q_{k}+A_{1}\left(S_{k} C_{k}^{-1} S_{k}\right)_{1} A_{1}^{\mathrm{T}}\right) u}{u^{\mathrm{T}}\left(I+A_{1}\left(\Delta_{k}\right)_{1}^{-1} A_{1}^{\mathrm{T}}\right) u} \\
& \leqslant \frac{u^{\mathrm{T}}\left(I+Q_{k}+A_{1}\left(\Delta_{k}\right)_{1}^{-1} A_{1}^{\mathrm{T}}\right) u}{u^{\mathrm{T}}\left(I+A_{1}\left(\Delta_{k}\right)_{1}^{-1} A_{1}^{\mathrm{T}}\right) u} \\
& \leqslant 1+\frac{u^{\mathrm{T}} Q_{k} u}{u^{\mathrm{T}} u} \\
& \leqslant 1+\left\|\left(C_{k}\right)_{2}^{-1}\right\|\left\|A_{2}\left(S_{k}\right)_{2}\right\|^{2} \\
& \leqslant 1+\frac{\left\|A_{2}\left(S_{k}\right)_{2}\right\|^{2}}{\tau}
\end{aligned}
$$

Let us observe that since the eigenvectors associated with unit eigenvalues satisfy

$$
\left(Q_{k}+A_{1}\left(S_{k} C_{k}^{-1} S_{k}\right)_{1} A_{1}^{\mathrm{T}}-A_{1}\left(\Delta_{k}\right)_{1}^{-1} A_{1}^{\mathrm{T}}\right) u=0
$$

the multiplicity of unit eigenvalues is equal to the dimension of the null space of matrix $Q_{k}+$ $A_{1}\left(S_{k} C_{k}^{-1} S_{k}\right)_{1} A_{1}^{\mathrm{T}}-A_{1}\left(\Delta_{k}\right)_{1}^{-1} A_{1}^{\mathrm{T}}$. Therefore, the existence of unit eigenvalues is not guaranteed.

It is worth comparing two possible systems solved by iterative methods: augmented system (55) that involves matrix $\mathscr{A}_{k}$ preconditioned with $\mathscr{P}_{k}$ given by (57) and normal equations (65) that involve matrix $\mathscr{F}_{k}$ preconditioned with $\mathscr{G}_{k}$ given by (66). The bounds on the spectra of the preconditioned matrices are given by Corollary 4.1 and Theorem 4.2 , respectively. For $\mathscr{P}_{k}^{-1} \mathscr{A}_{k}$ (with regularization given by (62)) from (59) and (63) we have

$$
1 \leqslant \lambda \leqslant 1+\frac{\left\|A_{2}\left(S_{k}\right)_{2}\right\|^{2}}{\tau}+\frac{1}{1-\tau}
$$

and for $\mathscr{G}_{k}^{-1} \mathscr{F}_{k}$ we have (67). We observe that for a practical choice of $\tau$ close to 0 the bounds are comparable. However, preconditioning of augmented system offers a slight advantage: first matrix $\mathscr{P}_{k}^{-1} \mathscr{A}_{k}$ is ensured to have a cluster of $m-n+n_{1}$ eigenvalues at one, second for $\tau$ very close 
to zero the bound of the ratio of the largest to the smallest eigenvalue of the preconditioned matrix is about two times smaller than that for preconditioned normal equations.

We conclude this section considering the limit case where the set $\mathscr{L}_{k}$ is empty. In this case, the linear system has form (39) where $\left\|S_{k}\right\| \leqslant 1-\tau$. To use a short-recurrence method, we can apply PCG to the normal system

$$
\left(S_{k}^{\mathrm{T}} A^{\mathrm{T}} A S_{k}+C_{k}\right) \tilde{p}_{k}=-S_{k} A^{\mathrm{T}}\left(A x_{k}-b\right)
$$

with preconditioner $S_{k} A^{\mathrm{T}} A S_{k}$. The application of the preconditioner can be performed solving a linear system with matrix

$$
\left(\begin{array}{cc}
I & A S_{k} \\
S_{k} A^{\mathrm{T}} & 0
\end{array}\right)
$$

\section{PRELIMINARY NUMERICAL RESULTS}

The numerical results were obtained in double precision using MATLAB 7.0 on a Intel Xeon (TM) 3.4 GHz, 1GB RAM. The threshold parameters were the same as in [3]: $\beta$ in (41) was set to 0.3 , $\theta$ in (43) and $\sigma$ in (17) were set to 0.9995 . The threshold $\tau$ in (51) was set to 0.1. A successful termination is declared when

$$
\left\{\begin{array}{l}
q_{k-1}-q_{k}<\varepsilon\left(1+q_{k-1}\right) \\
\left\|x_{k}-x_{k-1}\right\| \leqslant \sqrt{\varepsilon}\left(1+\left\|x_{k}\right\|\right) \\
\left\|P\left(x_{k}+g_{k}\right)-x_{k}\right\|<\varepsilon^{1 / 3}\left(1+\left\|g_{k}\right\|\right)
\end{array} \quad \text { or } \quad\left\|D_{k} g_{k}\right\| \leqslant \varepsilon\right.
$$

with $\varepsilon=10^{-9}$. A failure is declared when the above tests are not satisfied within 100 iterations. All tests were performed letting the initial guess $x_{0}$ be the vector of all ones.

First, we intend to investigate the effect of the regularization strategy on the conditioning of the augmented systems (39) and on the behaviour of the interior-point method. To this end, we monitor the 1-norm condition number of the arising augmented systems via the estimation provided by the MATLAB function condest and compare the following two choices of the regularization parameters (36)

$$
\begin{aligned}
& \delta_{k, i}=0, \quad i=1, \ldots, n \\
& \delta_{k, i}= \begin{cases}0 & \text { if } i \notin \mathscr{L}_{k} \\
\min \left\{\max \left\{10^{-3},\left(w_{k}\right)_{i}\left(e_{k}\right)_{i}\right\}, 10^{-2}\right\} & \text { otherwise }\end{cases}
\end{aligned}
$$

We remark that with choice (70) the augmented system is not regularized and the method reduces to the interior-point method given in [3]. On the other hand, choice (71) is in accordance with the results of Corollary 4.1. The safeguards used in the definition of $\delta_{k, i}$ avoid too small and too large regularization parameters.

The experiments are carried out on two sets of problems. The first set (Set 1 ) is made up of illc1033, illc1850, well1033, well1850 problems from the Harwell Boeing collection [18]. These problems are well-conditioned or moderately ill-conditioned and may be 
Table I. Names, dimensions, nonzero entries, minimum and maximum singular values of the matrices in Set 1 and set2.

\begin{tabular}{|c|c|c|c|c|c|c|c|}
\hline \multirow[b]{2}{*}{ Test name } & \multirow[b]{2}{*}{$m$} & \multirow[b]{2}{*}{$n$} & \multirow[b]{2}{*}{$n n z$} & \multicolumn{2}{|c|}{ Set 1} & \multicolumn{2}{|l|}{ Set2 } \\
\hline & & & & $\sigma_{1}$ & $\sigma_{n}$ & $\sigma_{1}$ & $\sigma_{n}$ \\
\hline illc1033 & 1033 & 320 & 4732 & $1.135 \times 10^{-4}$ & 2.144 & $6.801 \times 10^{-10}$ & 2.093 \\
\hline illc1850 & 1850 & 712 & 8758 & $1.511 \times 10^{-3}$ & 2.123 & $1.475 \times 10^{-9}$ & 1.845 \\
\hline wel11033 & 1033 & 320 & 4732 & $1.087 \times 10^{-2}$ & 1.807 & $1.682 \times 10^{-8}$ & 1.785 \\
\hline wel11850 & 1850 & 712 & 8758 & $1.612 \times 10^{-2}$ & 1.794 & $8.438 \times 10^{-8}$ & 1.683 \\
\hline
\end{tabular}

degenerate. In the second set (Set2), matrices and right-hand sides of the tests in Set1 are scaled by multiplying rows from index $n-1$ to index $m$ by a factor $16^{-5}$. This scaling was used in [19] and it gives rise to very ill-conditioned matrices with $\sigma_{1}$ close to 0 . In Table I, for each test we report the size of $A$, the number $n n z$ of nonzero entries of $A$, the minimum and maximum singular values $\sigma_{1}$ and $\sigma_{n}$ of $A$. Owing to the medium scale of these tests we solve the linear systems (39) via the MATLAB backslash operator.

Figures 1 and 2 show the condition number of the augmented system at each iteration of the interior point method applied to tests in Set1 and Set2. We compare the nonregularized augmented system (2) and the regularized (and reduced) system (55). Let us draw the reader's attention to the fact that the elimination of $C_{2}$ from (54) to produce system (55) does not noticeably change the condition number of the matrix. In other words, systems (54) and (55) have comparable conditioning. The improvement over the conditioning of (2) is due to the added regularization. Regarding tests in Set1, it is evident that if the problem is moderately ill-conditioned, the regularization produces a reduction, as expected, of the condition number of the augmented system; in fact, in the regularized case the condition numbers of matrices $A$ and $\mathscr{H}_{\delta}$ are comparable. On the other hand, when the problem is not ill-conditioned the regularization does not affect the condition number of the augmented system, see, e.g. wel11033 and wel11850 problems. Finally, the convergence history of the interior-point method does not seem to be affected by the introduction of the regularization strategy.

Analyzing the results of runs for test problems in Set2, we observe that these problems exhibit very ill-conditioned linear systems and the regularization strategy reduces their condition number by several orders of magnitude. The use of regularization in the interior-point method has improved significantly the performance of the solution of $i 11$ c1033 and illc1850. On the other hand, a failure occurred in the solution of wel11033 with and without regularization. Such failures are due to the selection of the scaled Cauchy step for a large number of iterations, which makes the method extremely slow.

Now, we intend to investigate the effectiveness of our preconditioning technique. We considered problems where matrix $A$ is the transpose of the matrices in the LPnetlib subset of The University of Florida Sparse Matrix Collection [20]. The vector $b$ is set equal to $b=-A e$, where $e$ is the vector of all ones. From LPnetlib collection we discarded the matrices with $m<1000$ and the matrices that are not full rank, getting a total of 56 matrices. When the 1-norm of $A$ exceeded $10^{3}$, we scaled the matrix using a simple row and column scaling scheme. Namely, we divide each row of $A$ by the geometric mean of the largest and the smallest nonzero element in this row, then we do the same for each column. The process is repeated twice. This technique has been used as a default scaling in HOPDM linear programming solver [21]. 

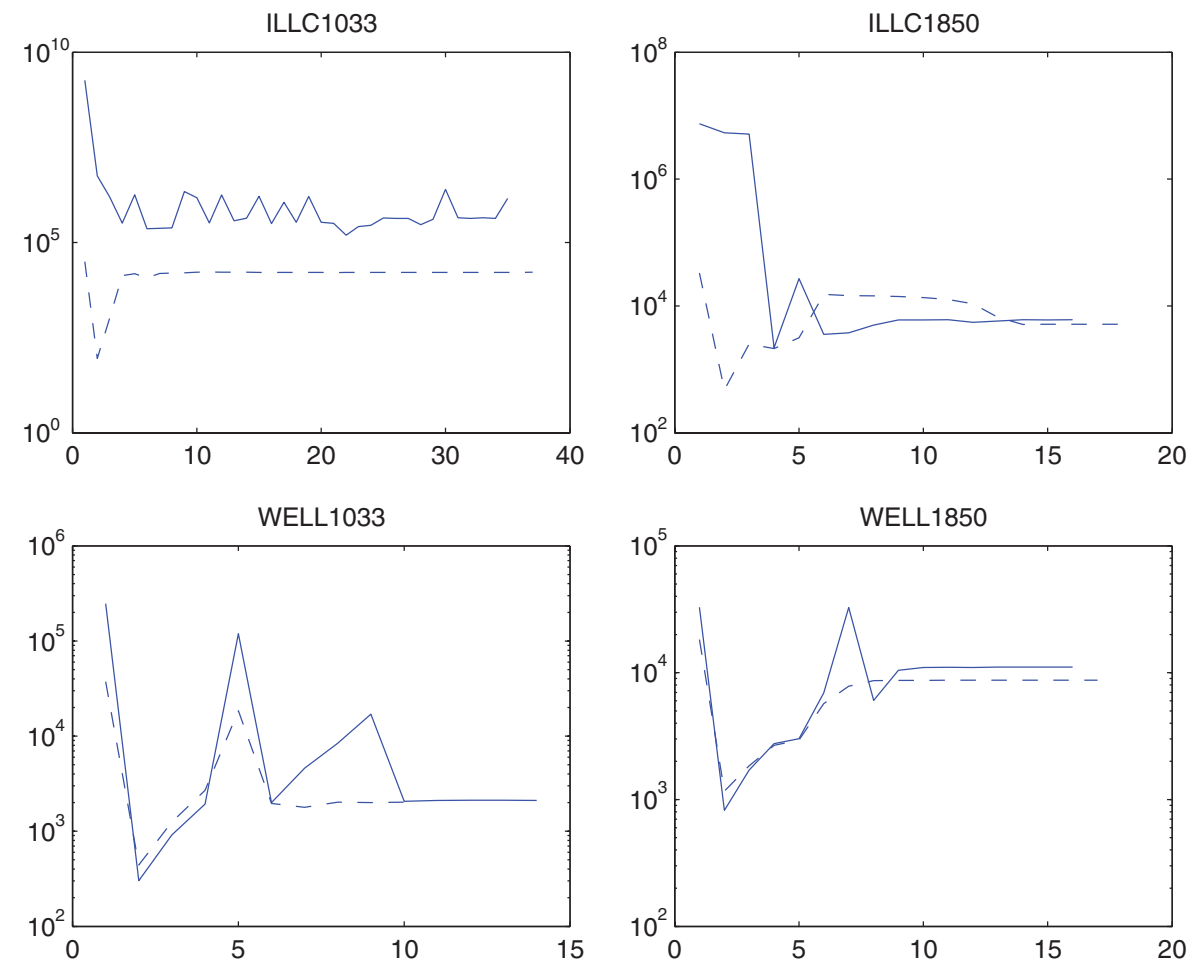

Figure 1. Condition number of the augmented systems arising in Set 1 with regularization (dashed line) and without regularization (solid line).

We use the iterative solver PPCG [8] and we set to 100 the maximal number of PPCG iterations. If PPCG was not able to satisfy the stopping criterion within 100 iterations, the algorithm employed the last computed iterate. Regarding the choice of the stopping tolerance for PPCG, a comment is needed. PPCG is an iterative procedure that yields the solution of the indefinite system (55) via a CG procedure for the symmetric positive system (65) preconditioned by $\mathscr{G}_{k}$ given in (66). Then, it provides a step $\tilde{p}_{k}$ such that

$$
\left(I+Q_{k}+A_{1}\left(S_{k} C_{k}^{-1} S_{k}\right)_{1} A_{1}^{\mathrm{T}}\right) \tilde{q}_{k}=-\left(A x_{k}-b\right)+\bar{r}_{k}
$$

and monitors the norm of preconditioned residual $\mathscr{G}_{k}^{-1 / 2} \bar{r}_{k}$. Letting

$$
\eta_{k}=\max \left(500 \varepsilon_{m}, \min \left(10^{-1}, 10^{-2}\left\|W_{k} D_{k} g_{k}\right\|\right)\right)
$$

we stop PPCG when $\left\|\mathscr{G}_{k}^{-1} \bar{r}_{k}\right\|$ drops below tol given by

$$
\text { tol }=\max \left(10^{-7}, \frac{\eta_{k}\left\|W_{k} D_{k} g_{k}\right\|}{\left\|A^{\mathrm{T}} S_{k}\right\|_{1}}\right)
$$

With this adaptive choice of tol, the linear systems are solved with a low accuracy when the current iterate is far from the solution, while the accuracy increases as the solution is approached. 

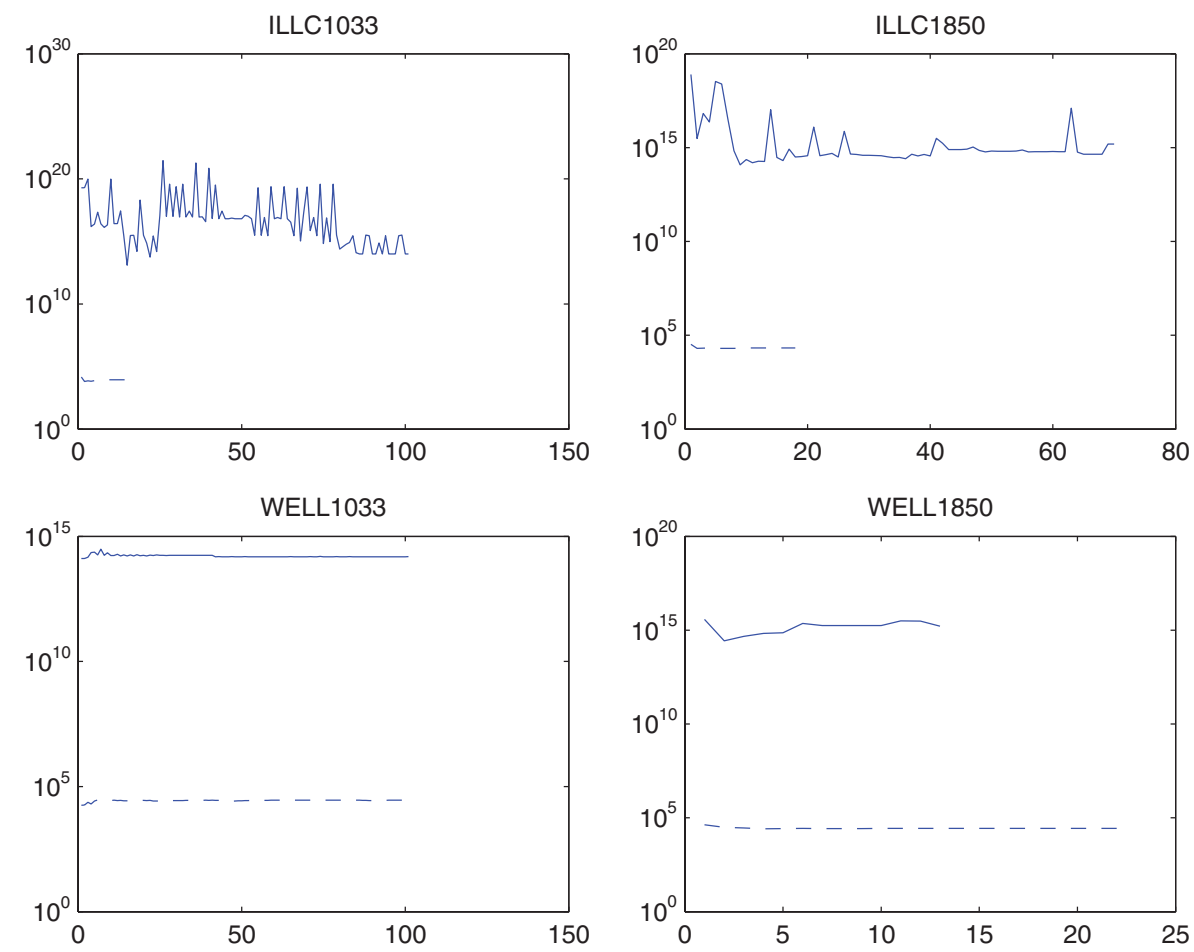

Figure 2. Condition number of the augmented systems arising in Set 2 with regularization (dashed line) and without regularization (solid line).

This choice allows one to solve with a sufficient accuracy also the linear system (38). Indeed, the unpreconditioned residual $\tilde{r}_{k}$ in (38) is related to the unpreconditioned residual $\bar{r}_{k}$ in (72) as follows:

$$
\bar{r}_{k}=S_{k}^{\mathrm{T}} A^{\mathrm{T}} \tilde{r}_{k}
$$

Then, with this choice of $t o l$ we enforce condition (16) with an $\eta_{k}$ sufficiently small to ensure quadratic convergence (see Theorem 3.1). Simpler choices of tol were not satisfying; for example, tol $=10^{-3}$ for all $k$ yields a very inaccurate solution of (38) that precludes the convergence of the method. On the other hand, tol $=10^{-7}$ for all $k$ yields an exceedingly accurate solution in the first iterations, and this requires many PPCG iterations as typically the preconditioner is not very efficient at the first iterations of the method.

When the set $\mathscr{L}_{k}$ is empty we use the CG method applied to the normal equations system without any preconditioner.

The regularization parameters $\delta_{k}$ have been chosen according to the rule (71). Moreover, to avoid preconditioner updates and factorizations, at iteration $k+1$ we freeze the set $\mathscr{L}_{k}$ and the vector $\delta_{k}$ if at $k$ th iteration PPCG has succeeded within 30 iterations and the following condition holds:

$$
\left|\operatorname{card}\left(\mathscr{L}_{k+1}\right)-\operatorname{card}\left(\mathscr{L}_{k}\right)\right| \leqslant 10
$$

Table II collects the results of the interior-point method on the performed runs. We report the problem name, the size of $A$, the number $n n z$ of nonzero elements of $A$, the number Nit of nonlinear 
Table II. Summary of the results for matrices from LPnetib.

\begin{tabular}{|c|c|c|c|c|c|c|c|c|}
\hline Test name & $m$ & $n$ & $n n z$ & Nit & Lit & $P f$ & ALit & $A v c$ \\
\hline lp_80bau3b & 12061 & 2262 & 23264 & 12 & 459 & 8 & 38 & 546 \\
\hline lp_bnl2 & 4486 & 2324 & 14996 & 10 & 566 & 8 & 57 & 747 \\
\hline lp_czprob & 3562 & 929 & 10708 & 6 & 7 & 0 & 1 & 0 \\
\hline lp_d2q06c & 5831 & 2171 & 33081 & $* *$ & $* *$ & $* *$ & $* *$ & $* *$ \\
\hline lp_degen 3 & 2604 & 1503 & 25432 & 21 & 1033 & 16 & 49 & 586 \\
\hline lp_dfl001 & 12230 & 6071 & 35632 & 10 & 417 & 7 & 42 & 829 \\
\hline lp_fffff 800 & 1028 & 524 & 6401 & 100 & 2384 & 94 & 24 & 69 \\
\hline lp_finnis & 1064 & 497 & 2760 & 15 & 588 & 10 & 39 & 149 \\
\hline lp_fit2d & 10524 & 25 & 129042 & 6 & 6 & 0 & 1 & 0 \\
\hline lp_fit $2 p$ & 13525 & 3000 & 50284 & 6 & 6 & 0 & 1 & 0 \\
\hline lp_ganges & 1309 & 1706 & 6937 & $* *$ & $* *$ & $* *$ & $* *$ & $* *$ \\
\hline lp_gfrd_pnc & 1160 & 616 & 2445 & 7 & 106 & 5 & 15 & 317 \\
\hline lp_ken_07 & 3602 & 2426 & 8404 & 12 & 122 & 6 & 10 & 2273 \\
\hline lp_ken_11 & 21349 & 14694 & 49058 & 14 & 255 & 6 & 18 & 14185 \\
\hline lp_ken_13 & 42659 & 28632 & 97246 & 12 & 172 & 8 & 14 & 27662 \\
\hline lp_ken_18 & 154699 & 105127 & 358171 & 11 & 156 & 7 & 14 & 101180 \\
\hline lp_maros & 1966 & 846 & 10137 & 13 & 500 & 7 & 38 & 323 \\
\hline lp_maros_r7 & 9408 & 3136 & 144848 & 6 & 6 & 0 & 1 & 0 \\
\hline lp_osa_07 & 25067 & 1118 & 144812 & 6 & 6 & 0 & 1 & 0 \\
\hline lp_osa_14 & 54797 & 2337 & 317097 & 6 & 6 & 0 & 1 & 0 \\
\hline lp_osa_30 & 104374 & 4350 & 604488 & 6 & 6 & 0 & 1 & 0 \\
\hline lp_osa_60 & 243246 & 10280 & 1408073 & 6 & 6 & 0 & 1 & 0 \\
\hline lp_pds_02 & 7716 & 2953 & 16571 & 10 & 121 & 4 & 12 & 2709 \\
\hline lp_pds_06 & 29351 & 9881 & 63220 & 10 & 192 & 6 & 19 & 9037 \\
\hline lp_pds_10 & 49932 & 16558 & 107605 & 10 & 215 & 9 & 22 & 15118 \\
\hline lp_perold & 1506 & 625 & 6148 & 34 & 1732 & 21 & 51 & 247 \\
\hline lp_pilot & 4860 & 1441 & 44375 & 13 & 760 & 10 & 58 & 501 \\
\hline lp_pilot4 & 1123 & 410 & 5264 & 15 & 597 & 8 & 40 & 98 \\
\hline lp_pilot87 & 6680 & 2030 & 74949 & 42 & 3839 & 40 & 91 & 914 \\
\hline lp_pilot_ja & 2267 & 940 & 14977 & 62 & 4154 & 50 & 67 & 461 \\
\hline lp_pilot_we & 2928 & 722 & 9265 & 12 & 662 & 9 & 55 & 359 \\
\hline lp_pilotnov & 2446 & 975 & 13331 & 44 & 2483 & 38 & 56 & 452 \\
\hline lp_qap8 & 1632 & 912 & 7296 & 7 & 11 & 3 & 2 & 11 \\
\hline lp_qap12 & 8856 & 3192 & 38304 & 7 & 11 & 3 & 2 & 17 \\
\hline lp_qap15 & 22275 & 6330 & 94950 & 7 & 11 & 3 & 2 & 21 \\
\hline 1p_scfxm2 & 1200 & 660 & 5469 & 76 & 2769 & 59 & 36 & 158 \\
\hline lp_scfxm3 & 1800 & 990 & 8206 & 84 & 3048 & 68 & 36 & 237 \\
\hline lp_scrs8 & 1275 & 490 & 3288 & 10 & 329 & 6 & 33 & 215 \\
\hline lp_scsd6 & 1350 & 147 & 4316 & 9 & 151 & 5 & 17 & 117 \\
\hline lp_scsd8 & 2750 & 397 & 8584 & $* *$ & $* *$ & $* *$ & $* *$ & $* *$ \\
\hline lp_sctap2 & 2500 & 1090 & 7334 & 8 & 68 & 4 & 9 & 10 \\
\hline lp_sctap3 & 3340 & 1480 & 9734 & 11 & 439 & 7 & 40 & 46 \\
\hline lp_shell & 1777 & 536 & 3558 & 7 & 68 & 1 & 10 & 528 \\
\hline lp_sierra & 2735 & 1227 & 8001 & 10 & 181 & 5 & 18 & 450 \\
\hline lp_standata & 1274 & 359 & 3230 & 7 & 107 & 5 & 15 & 67 \\
\hline lp_standmps & 1274 & 467 & 3878 & 10 & 216 & 4 & 22 & 94 \\
\hline 1p_stocfor2 & 3045 & 2157 & 9357 & 18 & 319 & 8 & 18 & 874 \\
\hline lp_stocfor3 & 23541 & 16675 & 72721 & $* *$ & $* *$ & $* *$ & $* *$ & $* *$ \\
\hline lp_truss & 8806 & 1000 & 27836 & 21 & 395 & 6 & 19 & 942 \\
\hline lp_wood $1 p$ & 2595 & 244 & 70216 & 22 & 656 & 14 & 30 & 204 \\
\hline lp_woodw & 8418 & 1098 & 37487 & 11 & 486 & 6 & 44 & 234 \\
\hline
\end{tabular}


Table II. Continued.

\begin{tabular}{|c|c|c|c|c|c|c|c|c|}
\hline Test name & $m$ & $n$ & $n n z$ & Nit & Lit & $P f$ & ALit & $A v c$ \\
\hline lpi_bgindy & 10880 & 2671 & 66266 & 7 & 19 & 3 & 3 & 23 \\
\hline lpi_ceria3d & 4400 & 3576 & 21178 & 9 & 94 & 2 & 10 & 287 \\
\hline lpi_klein3 & 1082 & 994 & 13101 & $* *$ & $* *$ & $* *$ & $* *$ & $* *$ \\
\hline lpi_cplex1 & 5224 & 3005 & 10947 & 12 & 266 & 9 & 22 & 1660 \\
\hline lpi_pilot4i & 1123 & 410 & 5264 & 14 & 638 & 8 & 46 & 95 \\
\hline
\end{tabular}

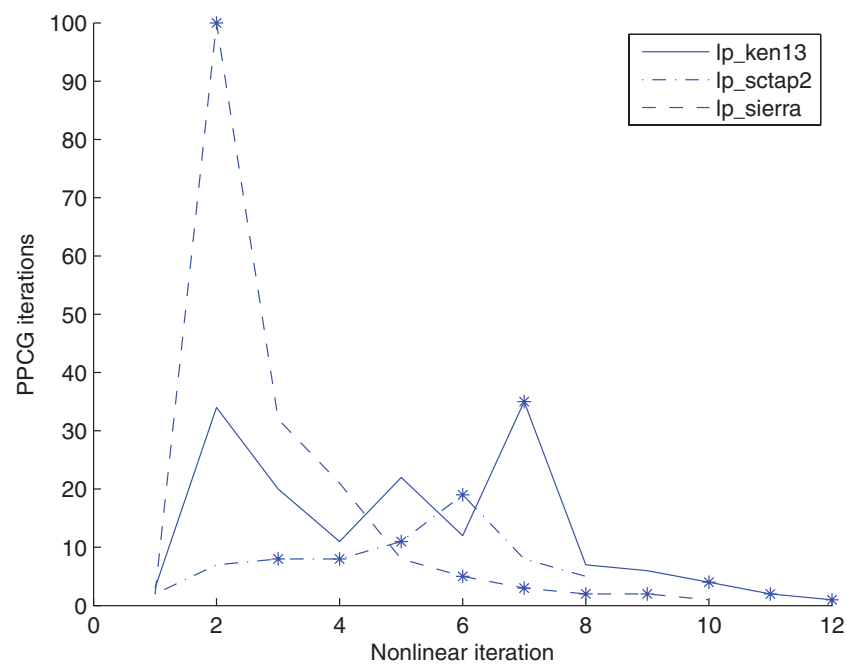

Figure 3. PPCG iterations against nonlinear iterations for 1p_ken13, 1p_sctap2, 1p_sierra.

iterations, the overall number Lit of PPCG iterations, the overall number Pf of preconditioner factorizations, the average number ALit of PPCG iterations and the average cardinality Avc of the set $\mathscr{L}_{k}$. On a total of 56 tests we have five failures in solving the problem within 100 nonlinear iterations; these runs are denoted by the symbol $* *$.

More insight into these failures, first we note that the linear algebra phase is effectively solved for all problems. Failures in problems $1 p_{-}$scsd 8 and $1 p_{-}$stocfor 3 are recovered allowing up to 300 nonlinear iterations. Specifically, Ip_scsd8 is solved with $N i t=201, L i t=1704, P f=8$, $A L i t=8, A v c=366$; 1p_stocfor3 is solved with $N i t=212, L i t=1956, P f=67, A L i t=9$ and

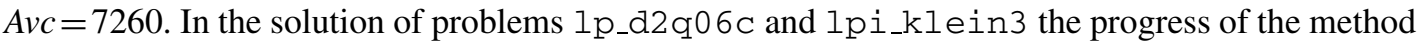
is very slow as the scaled Cauchy step is taken to update the iterates. On the contrary, in problem lp_ganges the projected Newton step is taken at each iterate but the procedure fails to converge in a reasonable number of iterations; we ascribe this failure to the use of the inexact approach as the Newton-like method with direct solver converges in eight iterations.

In Figure 3 we plot the number of PPCG iterations as the nonlinear iterations progress for 1p_ken13, 1p_sctap2 and 1p_sierra. These tests have been chosen as they display different behaviour and are representative enough for the general behaviour of the method. The symbol '*' 
in this figure indicates that at the corresponding iteration the factorization of the preconditioner was not needed and the set $\mathscr{L}_{k}$ and the vector $\delta_{k}$ have been frozen. As it can be seen from the plot, generally the number of PPCG iterations decreases when the sequence approaches the solution, despite the accuracy in the solution of the linear systems increases. This is due to the fact that in the final part of the iterative process the preconditioner works very well, as it is predicted by the theoretical analysis. In the case of $1 p_{\text {p_ken13 }}$ and 1p_sierra this excellent behaviour is obtained even if the set $\mathscr{L}_{k}$ and the vector $\delta_{k}$ have been frozen, because the set $\mathscr{L}_{k}$ settles down in the last iterations of the iterative process. On the other hand, 1p_sctap2 saved preconditioner factorizations in the middle of the iterative process, while close to the solution a great change in the set $\mathscr{L}_{k}$ forced the method to refresh the preconditioner. Finally, focusing on 1p_sierra, we can see that at the second iteration the method froze the set $\mathscr{L}_{k}$ and the regularization $\Delta_{k}$ and PPCG employed the maximum number of iterations allowed. Clearly, freezing was done prematurely at this stage and one should not allow it before the partition settles down.

We summarize these results.

- The interior-point method is robust and typically requires a low number of nonlinear iterations. On a total of 56 test problems we have five failures.

- The eight problems where $A v c$ is null are such that the solution is the null vector. In practice, for these problems we noted that $S_{k}$ is very small for all $k \geqslant 0$. Therefore, as $\Delta_{k}=0$, we have $S_{k}^{\mathrm{T}} A^{\mathrm{T}} A S_{k}+C_{k} \simeq I$ and the convergence of the linear solver is very fast. Therefore, we did not employ the preconditioner (69).

- There are noticeable savings in the number of preconditioner factorizations needed. Focusing on the 43 successfully solved test examples where the preconditioner was used, for 29 of them we avoided to update and factorize the preconditioner in at least $30 \%$ of the nonlinear iterations performed.

- For 32 out of 43 problems, $n_{1}$ is smaller than $n / 2$, that is, we have to solve augmented systems of considerably smaller dimension.

- The average number ALit of PPCG iterations is quite low. In the case of 40 out of 51 problems ALit does not exceed 40.

\section{CONCLUSIONS}

We have presented a new preconditioner for KKT systems arising in the regularized Newton-like method for solving NNLS problems. The preconditioner has been designed for the augmented system formulation of the KKT equation, which has grown from two observations: (i) the wellbehaved diagonal terms are eliminated from the equation to produce a reduced augmented system, (ii) the ill-behaved diagonal terms are regularized. As a result, the condition number of the reduced and regularized system is significantly smaller than that of the original KKT system. Such a reduced and regularized equation is easier to precondition and, ultimately, easier to solve by an iterative method.

We have performed the spectral analysis of the preconditioned matrix and have provided bounds on its condition number. These bounds do not depend on the interior-point scaling in the Newtonlike method. To the best of our knowledge it is the first result of this kind. We have implemented the method in MATLAB. Our preliminary computational results have confirmed all the theoretical findings. 


\section{ACKNOWLEDGEMENTS}

The visit of the second author to the University of Florence was partially supported by GNCS/INDAM, Italy.

\section{REFERENCES}

1. Björck A. Numerical Methods for Least Squares Problems. SIAM: Philadelphia, PA, 1996.

2. Lotstedt P. Perturbation bounds for the linear least-squares problem subject to linear inequality constraints. BIT 1983; 23:500-519.

3. Bellavia S, Macconi M, Morini B. An interior Newton-like method for nonnegative least-squares problems with degenerate solution. Numerical Linear Algebra with Applications 2006; 13:825-846.

4. Heinkenschloss M, Ulbrich M, Ulbrich S. Superlinear and quadratic convergence of affine-scaling interior-point Newton methods for problems with simple bounds without strict complementarity assumptions. Mathematical Programming 1999; 86:615-635.

5. Saunders M. Cholesky-based methods for sparse least-squares: the benefits of regularization. In Linear and Nonlinear Conjugate Gradient-related Methods, Adams L, Nazareth L (eds). SIAM: Philadelphia, PA, 1996; 92-100.

6. Altman A, Gondzio J. Regularized symmetric indefinite systems in interior point methods for linear and quadratic optimization. Optimization Methods and Software 1999; 11:275-302.

7. Benzi M, Golub GH, Liesen J. Numerical solution of saddle point problems. Acta Numerica 2005; 14:1-137.

8. Dollar HS, Gould NIM, Schilders WHA, Wathen AJ. Implicit-factorization preconditioning and iterative solvers for regularized saddle-point systems. SIAM Journal on Matrix Analysis and Applications 2006; 28:170-189.

9. Coleman TF, Li Y. An interior trust-region approach for nonlinear minimization subject to bounds. SIAM Journal on Optimization 1996; 6:418-445.

10. Dembo RS, Eisenstat SC, Steihaug T. Inexact Newton methods. SIAM Journal on Numerical Analysis 1982; 19:400-408.

11. Silvester D, Wathen A. Fast iterative solution of stabilised stokes systems. Part II: using general block preconditioners. SIAM Journal on Numerical Analysis 1994; 31:1352-1367.

12. Saunders M. Solution of sparse rectangular systems using LSQR and CRAIG. BIT 1995; 35:588-604.

13. van der Vorst HA. Bi-CGSTAB: a fast and smoothly converging variant of Bi-CG for the solution of nonlinear systems. SIAM Journal on Scientific and Statistical Computing 1992; 13:631-644.

14. Saad Y, Schultz MH. GMRES: a generalized minimal residual algorithm for solving nonsymmetric linear systems. SIAM Journal on Scientific and Statistical Computing 1986; 7:856-869.

15. Freund R, Nachtigal N. Software for simplified Lanczos and QMR algorithms. Applied Numerical Mathematics 1995; 19:319-341.

16. Dollar S. Iterative Linear Algebra for Constrained Optimization. Ph.D. Thesis, Oxford University Computing Laboratory, Keble College, University of Oxford, 2005.

17. Dollar HS, Gould NIM, Schilders WAH, Wathen AJ. Using constraint preconditioners with regularized saddle-point problems. Computational Optimization and Applications 2007; 36:249-270.

18. Duff I, Grimes R, Lewis J. Sparse matrix test problems. ACM Transactions on Mathematical Software 1989; 15:1-14.

19. Arioli M, Duff IS, de Rijk PPM. On the augmented system approach to sparse least-squares problems. Numerische Mathematik 1989; 55:667-684.

20. Davis TA. The University of Florida sparse matrix collection. NA Digest 1994; 92(42); 1996; 96(28); 1997; 97(23). Available from: http://www.cise.ufl.edu/research/sparse/matrices.

21. Gondzio J. HOPDM (version 2.12) — a fast LP solver based on a primal-dual interior point method. European Journal of Operational Research 1995; 85:221-225. 